\title{
An Improvement of the Nonparametric Repair-Limit Replacement Method by a Cubic Spline Approximation
}

\author{
T. Dohi ${ }^{\dagger}$, H. Nagai ${ }^{\dagger}$, N. Kaio ${ }^{\ddagger}$ and S. Osaki ${ }^{\dagger}$ \\ ${ }^{\dagger}$ Department of Industrial and Systems Engineering, Hiroshima University \\ 4-1 Kagamiyama 1 Chome, Higashi-Hiroshima 739-8527, JAPAN \\ dohi@gal.sys.hiroshima-u.ac.jp \\ $\ddagger$ Department of Economic Informatics, Hiroshima Shudo University \\ 1-1-1 Ozukahigashi, Asaminami-ku, Hiroshima 731-3195, JAPAN
}

\begin{abstract}
In this article, we consider a nonparametric repair-limit replacement (RLR) problem based on the scaled total time on test (TTT) statistics. It should be noted that the estimation procedure for the optimal repair-time limit via the scaled TTT statistics is made on the graph, but is not always stable when the sample size of repair time is small. In order to overcome this problem. wo estimate the optimal repair-time limit on an approximated scaled TTT curve. In other words, we apply a smoothing technique based on the natural cubic spline function to interpolate the scaled TTT curve.
\end{abstract}

\section{Introduction}

The concept of the scaled total time on test (TTT) statistics and the scaled TTT plot plays an important role to characterize the aging property of the lifetime distribution [1]. On the other hand, this concept is applicable to solve several kinds of optimal maintenance problems. Bergman [2] discovered this graphical solution method for the simple age replacement problem. Koshimae, Dohi, Kaio and Osaki [3] and Dohi, Matsushima, Kaio and Osaki [4] applied the similar technique to the repair-limit replacement (RLR) problems. More preciscly: in the elassical RLR problems to derive the optimal repair-time limit which minimizes the oxpereded cost pere unit time in the steady-state. the melerlying optimization problem ean be reduced to a graphical one on the sealed TTT emese siner the experted cost function (an be reperesented by the repair-tine distribution function and the eorresponding sealed TT transform.

On the other hand. when the empirical data of repair time are giren. the graphical method abore can be used to estimate the optimal repair-time limit. That is, replacing the scaled TTT curve into the scaled TTT plot, we can estimate the optimal policy without specifying the repair-time distribution function. In addition, notice that the nonparametric method based on the scaled TTT plot can guarantee the strongly consist estimator of the optimal repair-time limit. This property is powerful and has the advantage of practical use. In general, since the specification of the distribution from data is not easy, it will be useful for practitioners to determine the maintenance schedule without selection of the repairtime distribution.

If one has to be estimate the optimal repair-time limit from a small sample of repair-time data, howerer, the estimate is expected to be poor. Since the scaled TTT plot is a step function of the empirical distribution and the scaled TTT statistics, it can not represent the real (but unknown) scaled TTT curve from a small sample. This results from the fact that the scaled TTT plot is not analytic and is not always a suitable approximate function for the scaled TTT transform with unknown repair-time distribution. In this article, we propose a method to approximate the scaled TTT plot by an analytic function and attempt to estimate the optimal repair-time limit precisely from a small sample data. Then the natural cubic Spline function is used to interpolate the scaled TTT plot. In the following section, we describe the RLR problem. In Section 3, the TTT concept is introduced and the optimal RLR policy is derived on the scaled TTT curve. Section 4 is devoted to explain the empirical estimation method based on the TTT plot. In Section 5 , we apply a smoothing technicque based on the natural cubic spline function to interpolate the scaled TTT ('urve and propose an algorithm to estimate the optimal polier: Nunnerical illustrations are presented in Sertion 6. Finally: the papere conchudes with some remarks in Sertion 7 .

\section{The RLR Problem}

Consider a single unit system, where ach spare is provided only by an order after a lead time $L(>0)$ and each failed unit is repairable. The original unit begins operating at time 0 . The mean lifetime for each unit is $1 / \lambda(>0)$. When the unit has failed, the repair is started immediately. If the repair is completed up to the time limit for repair $t_{0} \in[0, \infty)$, then the unit is installed at 
that time. It is assumed that the unit once repaired is presumed as good as new. However, if the repair time is greater than $t_{0}$, i.e. the repair is not completed up to the time $t_{0}$, the repair is retired and the failed unit is scrapped. Then, the spare unit is ordered immediately and delivered after the lead time $L$. The time required for replacement is negligible for convenience. The repair time for each unit has an arbitrary distribution $G(t)$ with density $g(t)$ and finite mean $1 / \mu(>0)$, where the function $G(\cdot)$ is assumed to have an inverse function, i.e. $G^{-1}(\cdot)$, and to be absolutely continuous and strictly increasing. Without any loss of generality, we assume $G(0)=0$ and $\lim _{t \rightarrow \infty} G(t)=1$. Under these model assumptions, we define the time interval from the start of the operation to the following start as one cycle. The configuration of the RLR model under consideration is depicted in Fig. 1.
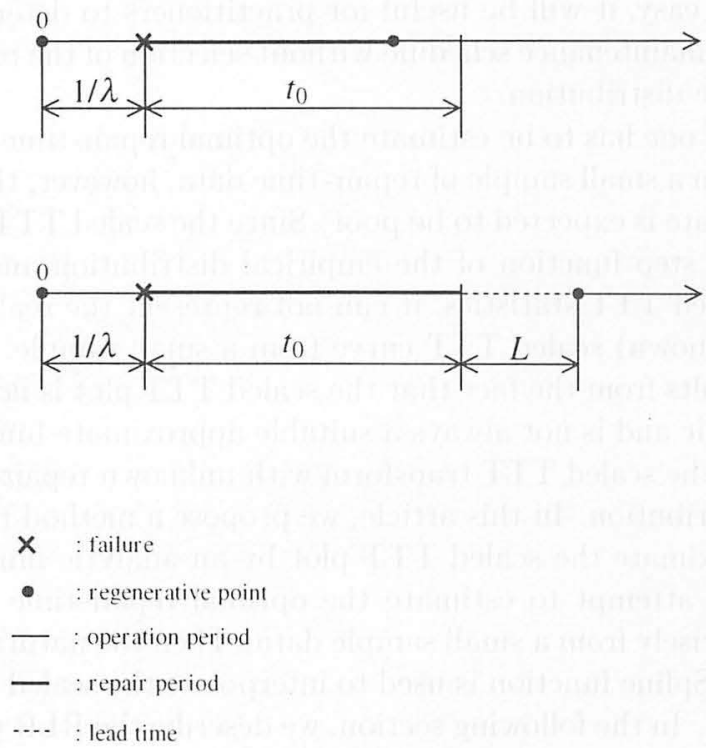

Fig. 1: The configuration of the RLR model.

Next. we consider the cost structure. The costs considered in this paper are the following:

$k_{r}(>0)$ : a cost per unit repair time

$h_{\text {s }}(>0)$ : a cost per minit shortage period

c $(>0)$ : an ordering cost for each spare unit.

We make the assumption:

$(\mathrm{A}-1) k_{r} L<c$

This assumption implies that the unit ordering cost is greater than the repair cost during the interval $[0, L]$, i.e. until the delivery of a new unit. For an infinite planning horizon, it will be appropriate to adopt an expected cost per unit time in the steady-state. Since the mean time of one cycle is

$$
\begin{aligned}
T\left(t_{0}\right) & =\int_{0}^{t_{0}}(1 / \lambda+t) d G(t)+\int_{t_{0}}^{\infty}\left(1 / \lambda+t_{0}+L\right) d G(t) \\
& =1 / \lambda+\int_{0}^{t_{0}} \bar{G}(t) d t+L \bar{G}\left(t_{0}\right)
\end{aligned}
$$

and the total expected cost for one cycle is

$$
V\left(t_{0}\right)=\left(k_{r}+k_{s}\right) \int_{0}^{t_{0}} \bar{G}(t) d t+\left(k_{s} L+c\right) \bar{G}\left(t_{0}\right),
$$

where $\bar{G}(t)=1-G(t)$, then the expected cost per unit time in the steady-state is, from the well-known renewal reward argument [5],

$$
\begin{aligned}
C\left(t_{0}\right) & \equiv \lim _{t \rightarrow \infty} \frac{[\text { the total cost on }(0, t]]}{t} \\
& =V\left(t_{0}\right) / T\left(t_{0}\right)
\end{aligned}
$$

and the problem is to determine the optimal repair-time limit $t_{0}{ }^{*}$ such as

$$
C\left(t_{0}^{*}\right)=\min _{0 \leq t_{0}<\infty} C\left(t_{0}\right) .
$$

It is straightforward to seek $t_{0}^{*}$ by differentiating $C\left(t_{0}\right)$ with respect to $t_{0}$, but we employ a different graphical method in the following section.

\section{The TTT Concept}

Before solving the problem in Eq.(4), we describe the total time on test (TTT) transform for the repair time distribution. Define the scaled total time on test (TTT) transform of the repair-time distribution $p \equiv G(t)$ by

$$
\phi(p) \equiv \mu \int_{0}^{G^{-1}(p)} \bar{G}(t) d t, \quad 0 \leq p \leq 1,
$$

where

$$
G^{-1}(p)=\inf \{t \geq 0: G(t) \geq p\} .
$$

The curve $\mathcal{L}=(p . \phi(p)) \in[0.1] \times F(0.1]$ is callod the scaled TTT transform or simply the scaled TTT carve. Wr shall propose a graphical method to solve the problem in Eq.(4) on the scalod TT T ("mrve.

The following result is due to Koshimae. Dohi. Kaio and ()saki [3].

Lemma 3.1: Suppose that the assumption (A-1) holds. The minimization problem in Eq.(4) is equiralent to obtain $p^{*}\left(0 \leq p^{*} \leq 1\right)$ satisfying

$$
\min _{0 \leq p \leq 1}: M(p, \phi(p)) \equiv \frac{\phi(p)+\xi}{p+\eta},
$$

where

$$
\xi \equiv \frac{\left(k_{s} L+c\right) \mu}{\left(c-k_{r} L\right) \lambda}>0
$$


and

$$
\eta \equiv-\left(1+\frac{k_{r}+k_{s}}{\left(k_{r} L-c\right) \lambda}\right)
$$

From Lemma 3.1, the optimal policy is $p^{*}=G\left(t_{0}^{*}\right)$ which minimizes the tangent slope from $(-\eta,-\xi)$ to the curve $\mathcal{L}$. Hence, it is straightforward to calculate $t_{0}^{*}=G^{-1}\left(p^{*}\right)$.

More precisely, we characterize the optimal policy from the aging property of $G(t)$.

Definition 3.2: (i) The repair-time distribution $G(t)$ is IRR (DRR) if and only if the instantaneous repair rate $r(t)=g(t) / \bar{G}(t)$ is increasing (decreasing).

(ii) $G(t)$ is IRR (DRR) if and only if $\phi(p)$ is concare (convex) in $p \in[0,1]$.

The relationship (ii) between the aging and the scaled TTT transform was proved by Barlow and Campo [1]. In the plane $(x, y)=(-\infty,+\infty) \times(-\infty,+\infty)$, define the following three points

$$
\begin{gathered}
\mathrm{B} \equiv\left(x_{B}, y_{B}\right)=(-\eta,-\xi), \\
\mathrm{Z} \equiv\left(x_{Z}, y_{Z}\right)=\left(\frac{\left(k_{s} L+c\right) r(0)}{\left(k_{r} L-c\right) \lambda},-\xi\right)
\end{gathered}
$$

and

$$
\mathrm{I} \equiv\left(x_{I}, y_{I}\right)=\left(-\eta .1+\frac{\left(k_{r}+k_{s}\right) \mu}{\left(k_{r} L-c\right) \lambda r(\infty)}\right) .
$$

Theorem 3.3: (1) Suppose that the scaled TTT curve $\mathcal{L}$ is strictly convex under the assumption $(\mathrm{A}-1)$.

(i) If $x_{B}>x_{Z}$ and $y_{B}>y_{I}$, then there exists a unique optimal solution $p^{*}=G\left(t_{0}^{*}\right)\left(0<t_{0}^{*}<\infty\right)$ minimizing the expected cost per unit time in the steadystate given by Eq. (3). where $p^{*}$ is giren by the $x$-coordinate in the point of contact for the curve $\mathcal{L}$ from the point B. where

$$
\max (0 .-1)<p^{*}<1 \text {. }
$$

(ii) If $x_{B} \leq x \%$. then the optinal repair-linnit policy is $p^{*}=(i(0)=0$.

(iii) If $y_{B} \leq y_{I}$. then the optimal reparir-linnit policy is $p^{*}=G(x)=1$.

(2) Suppose that the scaled TTT curve $\mathcal{L}$ is concare under the assumption (A-1). Then, the optimal solution is $p^{*}=0$ or $p^{*}=1$.

Proof: Differentiating $M(p, \phi(p))$ with respect to $p$ and setting it equal to zero implies

$$
q(p) \equiv \phi^{\prime}(p)(p+\eta)-(\phi(p)+\xi)=0,
$$

where

$$
\phi^{\prime}(p)=\frac{\mu}{r\left(G^{-1}(p)\right)}
$$

and the symbol' denotes the differentiation. Further, we have

$$
q^{\prime}(p)=\phi^{\prime \prime}(p)(p+\eta) .
$$

When the scaled TTT curve $\mathcal{L}$ is strictly convex, then $q^{\prime}(p)>0$ and the function $M(p, \phi(p))$ is strictly convex in $p$.

In the plane $(x, y) \in(-\infty,+\infty) \times(-\infty,+\infty)$, we define the point $\mathrm{B}=\left(x_{B}, y_{B}\right)$. Since the tangent line for the point $\left(p^{*}, \phi\left(p^{*}\right)\right)$ on the curve $\mathcal{L}$ is

$$
y=\frac{\mu}{r\left(G^{-1}\left(p^{*}\right)\right)}\left(p-p^{*}\right)+\phi\left(p^{*}\right),
$$

the condition that the point $B$ is on the above tangent line is $q\left(p^{*}\right)=0$. Define the intersection $\mathrm{Z}=\left(x_{Z}, y_{Z}\right)$ of the tangent line for the origin ()$=(0,0)$ on the curve $\mathcal{L}$ and $y=-\xi$. If the $x$-coordinate of $\mathrm{B}$ is strictly greater than the $x$-coordinate of $\mathrm{Z} . q(0)<0$, otherwise. $q(0) \geq 0$ under the assmmption (A-1). Similarly. define the intersection $\mathrm{I}=\left(x_{1}, y_{l}\right)$ of the tangent line for the point $\mathrm{U}=(1,1)$ on the $\mathcal{L}$ and $x=-\eta$. If the $y$-coordinate of $B$ is strictly greater than the $y$ - coordinate of $\mathrm{I}, q(1)>0$, otherwise. $q(1) \leq 0$ under $(\mathrm{A}-1)$. From these, we obtain the results (1).

Secondly, consider the "ase where $G(t)$ is IRR. In this case, $\phi(p)$ becomes a concave function of $p$. If the $x$ coordinate of $B$ is strictly negative and if the slope of the straight line BO is strictly smaller than that of the line BU. we have

$$
\left(k_{s} L+c\right) / \lambda-\left\{k_{r} L-c+\left(k_{r}+k_{s}\right) / \lambda\right\} / \mu<0,
$$

which is equiralent to the condition of $M(0, \phi(0))<$ $M(1, \phi(1))$. Conversely; if $x_{13}<0$ and if the slope of the straight line $\mathrm{BO})$ is not small than that of the line $\mathrm{BU}$, $M(0 . \phi(0)) \geq M(1 . \phi(1))$ is satisfied. On the other hand, the condition $x_{B} \geq 0$ implies $M(0 . \phi(0))>M(1 . \phi(1))$. Thus the proof is completed.

\section{An Empirical Method}

Based on the graphical ideas in Sertion 3. we propose a statistical mothod to estimate the optimal RLR policy. Suppose that the optimal repair-time limit has to be estimated from an ordered complete sample $0=x_{0} \leq x_{1} \leq$ $x_{2} \leq \cdots \leq x_{n}$ of repair times from an absolutely continuous repair-time distribution $G$, which is unknown. The estimator of $G(t)=p$ is the empirical distribution given by

$$
G_{n}(x)=\left\{\begin{array}{cc}
i / n & \text { for } x_{i} \leq x<x_{i+1} \\
1 & \text { for } x_{n} \leq x
\end{array}\right.
$$


$(i=0,1,2, \cdots, n-1)$. Then the scaled TTT statistics based on this sample are

$$
\phi_{i, n} \equiv S_{i} / S_{n}
$$

where $S_{0}=0$ and

$$
S_{i} \equiv \sum_{j=1}^{i}(n-j+1)\left(x_{j}-x_{j-1}\right), \quad i=1,2, \cdots, n .
$$

By plotting the point $\left(i / n, \phi_{i, n}\right),(i=0,1,2, \cdots, n)$, and connecting them by line segments, we obtain the socalled scaled TTT plot, $\mathcal{L}_{n} \in[0,1] \times[0,1]$.

As an empirical counterpart of Lemma 3.1, we propose a nonparametric estimator of the repair-time limit.

Theorem 4.1: The optimal repair-time limit can be estimated by $\hat{t_{0 n}}=x_{i^{*}}$, where

$$
\left\{i^{*} \mid \min _{0 \leq i \leq n} \frac{\phi_{i, n}+\xi}{i / n+\eta}\right\} \text {. }
$$

The proof is omitted for brevity:

For a better understanding of the theorem above, we give the following simple example.

Example 4.2: The repair-time data were made by the random number following the Weibull distribution with shape parameter $\alpha=0.2$ and scale parameter $\beta=2.0$. The other model parameters are $1 / \lambda=0.8000$ [day], $L=$ 0.2000 [day], $c=6.5000$ [\$], $k_{r}=4.0000$ [\$] and $k_{s}=$ $6.0000[\$]$. The scaled TTT plot based on the 200 sample data is shown in Fig. 2. Since $B=(-0.4035,-0.4771)$, the optimal point with minimum slope from $\mathrm{B}$ becomes $\left(i^{*} / n, \phi_{i^{*}, n}\right)=\left(119 / 200, \phi_{119,200}\right)=(0.5980,0.4873)$. Thus, the estimator of the optimal repair-time limit $\hat{t}_{0,200}=x_{119}=1.7006$.

Of our next interest is the investigation of asymptotic property of the estimator $\hat{t}_{0 n}$ in Theorem 4.1. The following theorem guarantees the astmptotic optimality of the estimator above.

Theorem 4.3: (i) The expected cost $C\left(t_{0 n}\right)$ of using the repair-time limit $\hat{t}_{0 n}$ tends with probability one to $C\left(t_{0}^{*}\right)$ as $n$ tends to infinity if $t_{0}^{*}$ is positive and finite.

(ii) The minimum expereted cost per unit time in the steady-state $C\left(t_{0}^{*}\right)$ may be estimated by

$$
\hat{C}\left(\hat{t}_{0 n}^{*}\right)=\frac{\left(k_{r}+k_{s}\right) o_{i, n}^{*} / \mu_{n}+\left(k_{s} L+c^{\prime}\right)\left(1-p_{n}^{*}\right)}{1 / \lambda+\phi\left(p_{n}^{*}\right) / \mu_{n}+L\left(1-p_{n}^{*}\right)}
$$

where $1 / \mu_{n}$ is the empirical mean of the repair time. Then the estimator is strongly consistent.

(iii) If a unique optimal repair-time limit exists then $\hat{t}_{0 n}$ is strongly consistent.

The results above can be seen from the analogies by Bergman [2]. However, it will be clear that the estimator $\mathcal{L}_{n}$ for $\mathcal{L}$ is very rough for the small sample data, since its asymptotic convergence rate is rather small experimentally. For instance, we need the number of data more than 100 to obtain a nice value closed to the real optimum. Hence, we propose to approximate $\phi_{i, n}$ by using the natural cubic spline function in the following section.

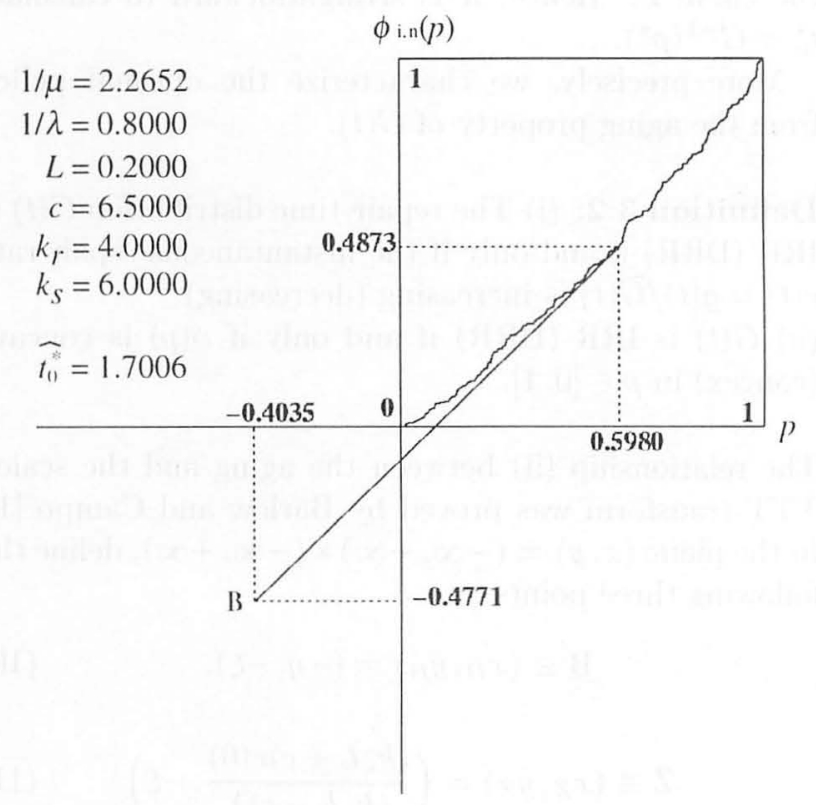

Fig. 2: Estimation of the optimal repair-time limit on the scaled TTT plot.

\section{The Spline Approximation}

During the last years, spline functions have found widespread application, mainly for the purpose of interpolation. The spline function is one of the piecewise continuous polynomials which is uscful to interpolate arbitrary discrete points so as to satisfy any continuous condition. In the two-climensional plane. let $x_{i}, y_{i}$. $i=0.1 . \cdots . \|$ be given and assume $x_{0}=0<x_{1}<$ $\cdots<r_{n}=1$ and $y_{0}=0<y_{1}<\cdots<y_{n}=1$. Then the function $z(x)$ is abled the spline function with order $m$. where $x=\left(x_{0} \ldots r_{1}, \cdots x_{n}\right)$. if $z(x)$ is the polynomial whose order is less than or equal to $m$ in the interval $\left[x_{;}, x_{i+1}\right](i=0.1 . \cdots . n-1)$ and is belonging to $\mathcal{C}^{m-1}$ (lass. Also. the spline function $z(x)$ is called the $X$ (natural)-spline function, if $z(x)$ with order $2 k-1$ $(k=1,2, \cdots)$ (an be represented by a polrnomial with order $k-1$ in the two intervals. $\left(-\infty, x_{1}\right]$ and $\left[x_{n}, \infty\right)$.

In general, it is well known that spline functions are better to approximate arbitrary functions than other polynomials with same number of parameters. Also, if we take account of the computational effort and precision in functional approximation, the natural cubic 
spline is probably the best candidate to use for interpolation. From the reason above, we approximate the scaled TTT plot by the natural cubic spline function. For $n+1$ points $\left(x_{i}, y_{i}\right)=\left(i / n, \phi_{i}\right),(i=0,1, \cdots, n)$ on the scaled TTT plot and $t \in\left[x_{i}, x_{i+1}\right]$, define the piecewise continuous polynomial with order three, $z_{i}(t)$, which is through the point $x_{i}$, as

$$
\begin{aligned}
z_{i}(t)= & \frac{\left(x_{i+1}-t\right)^{3}}{6 h_{i}} M_{i}+\frac{\left(t-x_{i}\right)^{3}}{6 h_{i}} M_{i+1} \\
& +A_{i}\left(x_{i+1}-t\right)+A_{i+1}\left(t-x_{i}\right),
\end{aligned}
$$

where

$$
h_{i}=x_{i+1}-x_{i}
$$

and

$$
A_{i}=\frac{y_{i}}{h_{i}}-\frac{h_{i}}{6} M_{i}, \quad i=0,1, \cdots, n-1 .
$$

In Eq. (24), the parameter $M_{i}(i=0,1, \cdots, n)$ is called the spline coefficient and satisfies the following simultaneous equations.

$$
\begin{gathered}
\frac{1}{4} M_{i-1}+M_{i}+\frac{1}{4} M_{i+1}=\frac{3\left(y_{i-1}-2 y_{i}+y_{i+1}\right)}{2 h_{i}^{2}}, \\
i=1,2, \cdots, n-1, \\
M_{0}+\frac{1}{2} \Lambda_{1}=\frac{3}{h_{i}}\left(\frac{y_{1}-y_{0}}{h_{i}}\right) . \\
M_{n-1}=\frac{y_{n}-2 y_{n-1}+y_{n-2}}{h_{i}^{2}} .
\end{gathered}
$$

Finally, we can determine the cubic spline function $z_{i}(t)$ $(i=0,1, \cdots, n-1)$ for all $t \in\left[x_{0}, x_{n}\right]=[0,1]$ with $M_{i}$ by solving the simultaneous equations numerically:

We develop an algorithm to estimate the optimal repair-time limit by approximating the scaled TTT plot.

(Step 1) Observe $n$ order statistics of the repair-time data $x_{1}, x_{2}, \cdots, x_{n}$.

(Step 2) Plot $\left(p_{i, n} \cdot o_{i, n}\right)=\left(i / \| \cdot o_{i}\right) . i=() .1 . \cdots . n$ on $R^{2} \in[(0.1] \times[0.1]$.

(Step 3) Calculate the Spline coefficients fiom Eys. $(2 \bar{\tau})-(29)$ and interpolate the points $\left(p_{i, n} .0 ; . . n\right.$ by the cubic X-Spline curve.

(Step 4) Derire the r-coordinate of the point to minimize the tangent slope from B to the N-Spline curve.

(Step 5) Interpolate the empirical distribution in Eq.(19) by the N-Spline curve and generate an approximated continuous distribution. Taking the inversion transform, seek the estimate of the optimal repair-time limit.

\section{$6 \quad$ Numerical Illustration}

In this section, we compare two estimation procedures in terms of predictive ability. Figure 3 shows respective estimates of the optimal repair-time limit for two methods, where we distinguish the existing method and the approximated method developed in Section 5. From this figure, it is observed that New Method can give the better estimation performance than Existing one and that both estimates tend to take the same value as the number of repair-time data increases. Roughly speaking, two methods provide the similar result for more than 26 data. In Fig. 4, we examine the behavior of the estimate for minimum expected cost value. Comparing with Fig. 3, it is concluded that New Method can estimate the corresponding cost value precisely than Existing one, even if the number of data becomes large.

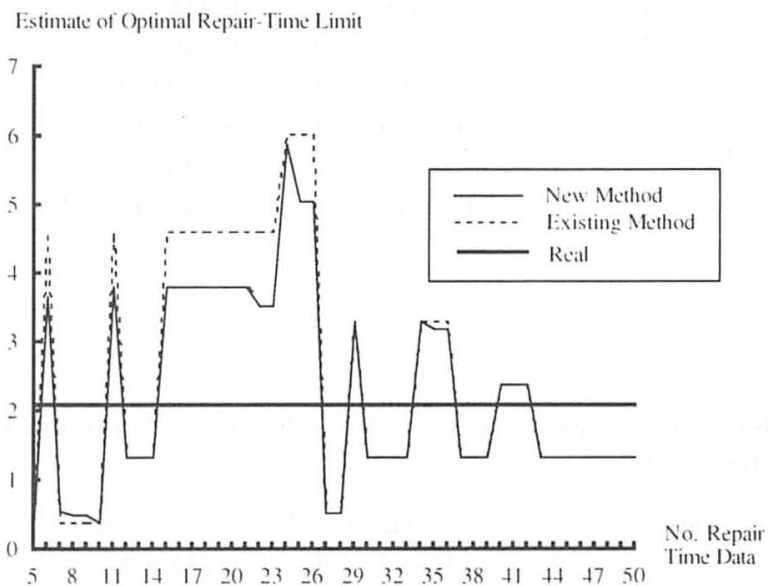

Fig. 3: Beharior of the Estimate for Optimal Repair-Time Limit.

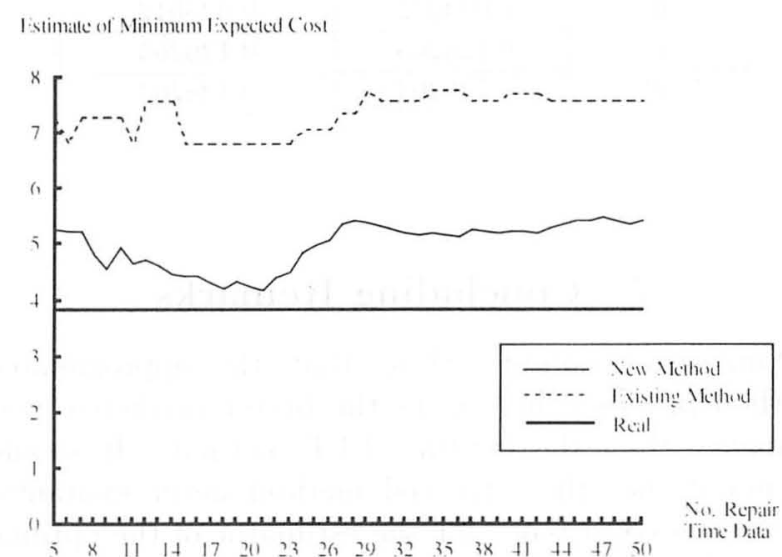

Fig. 4: Behavior of the Estimate for Minimum Expected Cost

In order to investigate the dependence of the number of repair-time data in estimates, we calculate the 
mean squared error between estimates and the real value for the optimal repair-time limit and the minimum expected cost in Tables 1 nd 2, respectively, using the different data from Figs. 3 and 4. From these results, we conclude that the method based on the Spline approximation can be recommended to estimate the optimal repair-time limit, especially for the small sample problem.

Table 1: Comparison of Two Methods for the Optimal Repair-Time Limit.

\begin{tabular}{|c||c||c|}
\hline No. Data & New Method & Existing Method \\
\hline 5 & 0.076178 & 0.224651 \\
\hline 10 & 0.051879 & 0.227414 \\
\hline 15 & 0.019075 & 0.194286 \\
\hline 20 & 0.009594 & 0.194286 \\
\hline 25 & 0.055516 & 0.211917 \\
\hline 30 & 0.081512 & 0.246708 \\
\hline 35 & 0.069092 & 0.259429 \\
\hline 40 & 0.075008 & 0.255393 \\
\hline 45 & 0.089604 & 0.246708 \\
\hline 50 & 0.089478 & 0.246708 \\
\hline
\end{tabular}

Table 2: Comparison of Two Methods for the Minimum Expected Cost.

\begin{tabular}{|c||c||c|}
\hline No. Data & New Method & Existing Method \\
\hline 5 & 0.711362 & 0.711362 \\
\hline 10 & 0.652048 & 0.652048 \\
\hline 15 & 0.678579 & 1.472546 \\
\hline 20 & 0.678579 & 1.472546 \\
\hline 25 & 0.894121 & 0.894121 \\
\hline 30 & 0.126263 & 0.126263 \\
\hline 35 & 0.292141 & 0.349452 \\
\hline 40 & 0.023012 & 0.023012 \\
\hline 45 & 0.126263 & 0.126263 \\
\hline 50 & 0.126263 & 0.126263 \\
\hline
\end{tabular}

\section{Concluding Remarks}

Numerical examples show that the apploximated method proposed here gives the better predictive performane than the existing TTT method. It should be noted that the improved method never guarantes the strong consistency for the estimator of the optimal repair-time limit, but is useful for the small sample problem which is an important problem in actual maintenance engineering practice.

Acknowledgment: This work was partially supported by a Grant-in-Aid for Scientific Research from the Min- istry of Education, Sports, Science and Culture of Japan under Grant No. 09780411 and No. 09680426.

\section{References}

[1] Barlow, R. E. and Campo, R., "Total time on test processes and applications to failure data analysis", in Reliability and Fault Tree Analysis, (eds. by R. E. Barlow, J. Fussell and N. D. Singpurwalla), 451481, SIAM, Philadelphia, (1975).

[2] Bergman, B., "On age replacement and the total time on test", Scandinavian Journal of Statistics, vol. 6, 161-168, (1979).

[3] Koshimae, H., Dohi, T., Kaio, N. and Osaki, S., "Graphical/statistical approach to repair limit replacement problem", Journal of the Operations Research, Society of Japan, vol. 39, 230-246, (1996).

[4] Dohi, T., Matsushima, N., Kaio, N. and Osaki, S., "Nonparametric repair-limit replacement policies with imperfect repair", European Journal of Operational Research, vol. 96, 260-273, (1996).

[5] Ross, S. M., Applied Probability Models with Optimization Applications, Holden-Day, San Francisco, (1970). 